\title{
Estudo comparativo de éguas repetidoras ou não de cio através da avaliação histológica do endométrio e das concentrações plasmáticas de progesterona ${ }^{1}$
}

\author{
Joana F. Eigenheer-Moreira ${ }^{2 *}$, Flávio T. Fernandes ${ }^{3}$, Fernando José R. Queiroz ${ }^{4}$, Tânia \\ G. Pinho ${ }^{5}$ e Ana Maria R. Ferreira ${ }^{6}$
}

\begin{abstract}
Eigenheer-Moreira J.F., Fernandes F.T., Queiroz F.J.R, Pinho T.G.\& Ferreira A.M.R. 2007. [Comparative study of repeat breeds and healthy mares through endometrial histology and plasmatic progesterone concentrations.] Estudo comparativo de éguas repetidoras ou não de cio através da avaliação histológica do endométrio e das concentrações plasmáticas de progesterona. Pesquisa Veterinária Brasileira 27(12):506-512. Curso de PósGraduação em Clínica e Reprodução Animal, Rua Vital Brazil Filho 64, Niterói, RJ 24230-340, Brazil. E-mail: joana.vet@gmail.com.br

The study aimed to compare endometrial histology and plasmatic progesterone ( $\mathrm{P} 4)$ concentration of repeat breeds and healthy mares. The hypothesis was that there is a correlation between infertility and endometrial histology and P4 concentration in both groups. A total of 36 Campolina and Mangalarga Marchador mares in reproductive age (3-23 years) were used, 11 of them were healthy mares (Control group, 7 embryo recipient and 4 embryo donors), and 25 repeat breeders (10 embryo recipient and 15 embryo donors), classified as based on their reproductive history. Endometrial and blood samples were collected for respectively histological and plasma progesterone concentration evaluation. The endometrial samples obtained after biopsy were fixed in Bouin's fluid, processed, included in paraffin, and stained with HematoxylinEosin (HE) for histopathological examination. Plasmatic progesterone concentrations were evaluated by enzyme immunoessay (ELISA). There was no correlation between progesterone concentration and fertility. But there was a positive correlation between age and fertility, as older mares had major tendency of subfertility than younger ones. There was also a correlation between biopsy categories and fertility, as more histological alterations were found, higher were the chances for the mares to be subfertile. However not all mares classified as Category I and II maintained pregnancy until parturition. Other factors could influence pregnancy maintenance. In the same way, not all mares in Category III were infertile. The endometrial biopsy was shown to be an easy and cheap diagnostic technique with minimal discomfort to the animals and, together with other data, to be a very important component in the investigation of mare fertility.
\end{abstract}

INDEX TERMS: Mare, subfertility, endometrial biopsy, histopathology, progesterone.

\footnotetext{
${ }^{1}$ Recebido em 19 de junho de 2007.

Aceito para publicação em 3 de dezembro de 2007.

${ }^{2}$ Curso de Pós-Graduação em Clínica e Reprodução Animal, Universidade Federal Fluminense (UFF), Rua Vital Brasil 64, Niterói, RJ 24320-340. *Autor para correspondência: joana.vet@gmail.com

${ }^{3}$ Médico Veterinário autônomo, Estrada da Granada 11, Papucaia, Cachoeiras de Macacu, RJ 28695-000, Brasil. E-mail: cral@ig.com.br

${ }^{4}$ Médico Veterinário autônomo, Estrada do Pacheco s/n, Km 12, Itaboraí, RJ 24800-000, Brasil. E-mail: equusveterinaria@globo.com

${ }^{5}$ Departamento de Patologia e Clínica Veterinária, Universidade Federal Fluminense (UFF), Rua Vital Brazil Filho 64, Niterói, RJ 24230-340, Brasil. Email: e.fuchs@terra.com.br

${ }^{6}$ Departamento de Patologia e Clínica Veterinária, UFF, Niterói, RJ. Pesquisadora do Conselho Nacional de Desenvolvimento Científico e Tecnológico 1C (CNPq). E-mail: anamrferreira@br.inter.net
}

RESUMO.- O trabalho teve como objetivo geral fazer um estudo comparativo da histologia endometrial e das concentrações plasmáticas de progesterona ( $\mathrm{P} 4)$ em éguas, repetidoras ou não de cio. A hipótese do presente estudo é que existe correlação entre o histórico de infertilidade, os achados histopatológicos e a concentração de $\mathrm{P} 4$ destes animais. Para tanto, foram utilizadas 36 éguas, em idade reprodutiva (3-23 anos), das raças Mangalarga Marchador e Campolina. Utilizaram-se éguas não repetidoras $(\mathrm{n}=11)$ como grupo controle, sendo 4 éguas doadoras e 7 receptoras; e repetidoras de cio $(n=25), 15$ doadoras e 10 receptoras. Tal classificação foi realizada de acordo com o histórico reprodutivo destes animais. Amostras de endométrio foram coletadas para a realização de avaliação histopatológica 
e amostras de sangue para a mensuração das concentrações plasmáticas de progesterona. Os fragmentos de tecido endometrial obtidos pela biópsia foram fixados no Fixador de Bouin. Posteriormente os fragmentos foram processados e incluídos em parafina. Os cortes foram corados por HematoxilinaEosina (HE) para exame histopatológico. As concentrações plasmáticas de $\mathrm{P} 4$ foram mensuradas pelo método de enzimaimunoensaio (ELISA). Não foi observada correlação entre as concentrações de P4 e a subfertilidade, sendo estas variáveis tratadas como independentes. Houve uma correlação positiva entre a idade e a subfertilidade $(\mathrm{p}<0.05)$, assim, quanto mais velha a égua, maior a subfertilidade. Foi observada uma correlação entre a categoria da biópsia e a subfertilidade $(\mathrm{p}<0.05)$, ou seja, quanto mais alterações histológicas, maiores as chances da égua ser subfértil. Contudo, nem todas as éguas classificadas na Categoria I e II levaram a gestação a termo e nem todas da Categoria III tiveram falhas na reprodução, pois, deve-se considerar que outros fatores podem influenciar na manutenção da gestação. A biópsia endometrial demonstrou ser uma técnica fácil, segura, barata e com um desconforto mínimo para o animal. Sugere-se que esta técnica, juntamente com outros dados, constitui uma importante ferramenta para a avaliação da fertilidade da égua.

TERMOS DE INDEXAÇÃO: Égua, subfertilidade, biópsia endometrial, histopatologia, progesterona.

\section{INTRODUÇÃO}

O emprego da transferência de embriões (TE) gerou um mercado crescente na indústria eqüina. Tal biotécnica possibilita um maior aproveitamento do potencial genético e reprodutivo de animais de alta performance.

A subfertilidade na égua constitui um dos maiores problemas na criação industrial equiina, causando grande perda econômica por impossibilitar a produção de um potro por ano (Concha-Bermejillo \& Kennedy 1982). Um dos aspectos mais importantes para que se mantenha a gestação é a existência de um útero sem alterações anatômicas e histológicas e particularmente um endométrio sadio (Kenney 1978). Desta forma, o diagnóstico de subfertilidade em éguas reprodutoras torna-se de extrema importância na tentativa de prevenir ou minimizar tais perdas econômicas.

$\mathrm{Na}$ espécie eqüina, independentemente do método de cobertura, o sêmen é depositado no lúmen uterino uma vez que a ejaculação ocorre em jatos. Tal evento decorre das características anatômicas da cérvix, que se encontra aberta durante o estro, e pela sua completa adaptação ao processo uretral, exposto durante a ereção da glande. Portanto, durante a cobertura as barreiras físicas são ultrapassadas, sendo os espermatozóides, proteínas do plasma seminal e bactérias do sêmen e do pênis do garanhão, responsáveis pela indução de uma resposta inflamatória aguda. Algumas éguas são mais susceptíveis e caracterizam-se pela sua incapacidade de eliminar o processo inflamatório em até 48 horas após a cobertura. De um modo geral, éguas susceptíveis apresentam características em comum, como idade avançada, histórico de falha reprodutiva em várias temporadas, histórico de episó- dios anteriores de endometrite e de perdas gestacionais (Troedsson 1997). No programa de TE, o trato reprodutivo da égua é manipulado também no diestro, momento em que se realiza a lavagem uterina para obtenção dos embriões nas doadoras e transferência do embrião nas receptoras. Durante tal período, com altas concentrações de progesterona que caracterizam o diestro, a cérvix encontra-se fechada e a contratilidade miometrial passa a apresentar longos períodos de contração, com baixa amplitude, o que caracteriza o tônus uterino típico da égua nesta fase. Todos estes fatores fazem com que a égua em diestro apresente uma menor capacidade de eliminação de uma possível contaminação e inflamação uterina (Evans et al. 1987, Jones et al. 1991). Assim, assume-se que éguas utilizadas em programas de TE estão mais predispostas a desenvolver endometrites.

A biópsia endometrial associada ao exame histopatológico proporciona informações importantes sobre as condições do endométrio, permitindo uma correlação entre os achados histopatológicos e a futura perspectiva da performance reprodutiva destas fêmeas (Queiroz 1991). A biópsia endometrial oferece como vantagens ser uma técnica relativamente fácil, segura (Ricketts 1975b, Doig et al. 1981, Kenney \& Doig 1986, Van Camp 1988) que pode ser realizada com um mínimo de equipamento e não está associada com dor ou desconforto do animal (Ricketts 1975b). Considerando que a P4 conduz ao estabelecimento e manutenção da gestação, a mensuração das concentrações plasmáticas desse hormônio também constitui uma importante forma de avaliação da performance reprodutiva das éguas (Cooneely 2001).

Este trabalho teve como objetivos comparar as características histopatológicas endometriais e as concentrações plasmáticas de P4 de éguas não repetidoras e repetidoras de cio. Este estudo poderá auxiliar no esclarecimento de tal problema reprodutivo e conseqüientemente, ajudar na realização de diagnósticos mais precisos, visando aumentar o potencial reprodutivo da espécie.

\section{MATERIAL E MÉTODOS}

\section{Animais}

Foram utilizadas 11 éguas não repetidoras de cio como grupo controle, 4 doadoras e 7 receptoras, determinadas como sadias e 25 éguas repetidoras de cio, 15 doadoras e 10 receptoras, classificadas como subférteis. Foram utilizadas éguas em idade reprodutiva (3-23 anos), das raças Mangalarga Marchador e Campolina, procedentes de duas Clínicas de Reprodução Equiina, sediadas no município de Itaboraí e Papucaia, ambas no Estado do Rio de Janeiro. Este projeto de pesquisa foi aprovado pelo Comitê de Ética em Pesquisa da Faculdade de Medicina da UFF/RJ, Hospital Universitário Antônio Pedro (CEP CMM/ HUAP no.111/06). As amostras destinadas aos exames histopatológicos e a mensuração das concentrações plasmáticas de P4 foram obtidas no período de setembro de 2005 a março de 2006.

\section{Delineamento experimental}

Classificação das éguas como repetidoras ou não de cio. Foram consideradas doadoras de embriões sadias aquelas das quais foi obtido embrião na lavagem uterina em todos os três últimos ciclos $(>3$ embriões). Quando não houve recuperação de embrião em cada um dos últimos três ciclos consecutivos, a égua foi classificada como doadora repetidora de cio ( $<3$ embriões). A coleta do embrião foi 
realizada no dia 7 ou 8 pós-ovulação. Após uma rigorosa limpeza da genitália externa e região perineal, um catéter de dupla via foi introduzido através do balão inflado com $20 \mathrm{~mL}$ de volume de ar. Foi infundido então, 1 litro da solução Ringer com Lactato na cavidade uterina, seguida por massagem uterina através do reto. A solução infundida foi drenada, com auxílio da gravidade, para um copo coletor com um filtro ligado a uma das extremidades do catéter. Este processo foi repetido mais uma vez. Após a lavagem uterina, o filtro ainda com uma pequena quantidade da solução do lavado uterino, foi levado até uma lupa para que pudesse ser feita a localização do embrião. $\mathrm{O}$ embrião identificado foi então transferido para uma placa de Petri e submetido a sete lavagens sucessivas em meio de manutenção do embrião ${ }^{7}$. Depois da realização deste processo, foram obtidas amostras de tecido endometrial, através de biópsia endometrial, assim como amostra de sangue para a mensuração das concentrações plasmáticas de progesterona. As éguas selecionadas para serem receptoras foram classificadas como não repetidoras de cio (sadias) utilizando-se como referência a ocorrência de gestação na estação anterior e ausência de alterações relevantes para a reprodução na avaliação ginecológica através da palpação e ultra-sonografia (US) do útero e dos ovários desses animais. Na ocorrência de uma gestação sem problemas na estação anterior, útero e ovários íntegros, sem alterações perceptíveis à palpação e à visualização na US, com características próprias da fase do ciclo estral, as éguas eram classificadas como sadias. As éguas classificadas como receptoras subférteis foram aquelas que não ficaram gestantes após três transferências de embrião consecutivas. No dia da transferência foi realizada a biópsia endometrial para avaliação histopatológica, assim como a coleta de sangue para a mensuração das concentrações plasmáticas de progesterona.

Coletas de sangue e mensuração de progesterona. Nas doadoras as coletas de sangue foram realizadas após a coleta do embrião, entre os dias 8 e 12 pós-ovulação. Nas receptoras, as coletas foram realizadas entre os dias 2 e 12 pós-ovulação. As amostras de sangue foram obtidas através da punção da veia jugular, utilizandose tubos a vácuo providos de anticoagulante (EDTA) e agulhas calibre $25 \times 8 \mathrm{~mm}$. Após a colheita, o sangue foi centrifugado a 3000rpm/ $10 \mathrm{~min}$. O plasma foi armazenado a $-20^{\circ} \mathrm{C}$ para posterior dosagem hormonal. As concentrações de $\mathrm{P} 4$ foram mensuradas pelo método de enzimaimunoensaio (ELISA), segundo procedimento recomendado pelo kit comercial empregado (EIAgen Progesterone, LI4010K, ADALTIS, Itália S.p.A). Todas as amostras foram mensuradas em um único ensaio. As soluções de referência de progesterona utilizadas no ensaio foram: Calibrador A: $0 \mathrm{ng} / \mathrm{mL}, \mathrm{B}: 0,25 \mathrm{ng} / \mathrm{mL}, \mathrm{C}: 1 \mathrm{ng} / \mathrm{mL}, \mathrm{D}$ : 2,5ng/mL, E: $5 \mathrm{ng} / \mathrm{mL}, \mathrm{F}: 10 \mathrm{ng} / \mathrm{mL}$ G: $20 \mathrm{ng} / \mathrm{mL}, \mathrm{H} 40 \mathrm{ng} / \mathrm{mL}$. O coeficiente de variação intra-ensaio foi de $10,7 \%$.

Biópsia endometrial. $\mathrm{O}$ fragmento da biópsia foi retirado com uma pinça tipo "boca de jacaré" (Biotech, Botucatu), de tamanho 20x4x3mm e comprimento total de $50 \mathrm{~cm}$. Esse instrumento possibilita a remoção de um fragmento representativo do endométrio. A coleta do fragmento foi realizada segundo procedimento descrito por Kenney \& Doig (1986). O fragmento, de aproximadamente $2 \mathrm{~cm}$, foi retirado da junção do corpo com o corno direito ou esquerdo, quando não havia nenhuma anormalidade no exame. Depois, foi acondicionado em solução de Bouin por 2 a 24 horas. Posteriormente, o fragmento foi lavado em soluções consecutivas crescentes de álcool (Kenney 1978, Kenney \& Doig 1986, Silva et al. 1987, Queiroz 1991). Em seguida os fragmentos foram submetidos ao clareamento em xilol, impregnação com parafina fundida, inclusão em molde retangular contendo parafina fundida sendo, por fim, corados com Hematoxilina-Eosina (HE), segundo metodologia descrita por

\footnotetext{
${ }^{7}$ IcpBioEMCARE, Embryo holding solution.
}

Junqueira \& Carneiro (1999). Para a avaliação histopatológica do endométrio foi utilizada a classificação de Kenney \& Doig (1986). Esta classificação inclui quatro categorias (I, IIA, IIB e III) que definem padrões, tipos e graus de gravidade das alterações inflamatórias e fibróticas do endométrio. Também correlaciona a categoria e o índice de fertilidade esperado (IFE) da égua. Segundo Kenney \& Doig (1986), na Categoria I o endométrio encontra-se normal ou com anormalidades muito leves e o IFE é de 80 a $90 \%$. Na categoria IIA são observadas alterações inflamatórias leves a moderadas, infiltração difusa no estrato compacto ou freqüentes focos inflamatórios dispersos no estrato compacto e no estrato esponjoso. Pode-se notar também freqüentes alterações fibróticas dispersas associadas a ramos individuais de glândulas de qualquer nível de severidade ou menos que dois ninhos glandulares fibróticos por $5,5 \mathrm{~mm}$ de campo linear em quatro ou mais campos; lacunas linfáticas evidentes ou atrofia endometrial parcial no fim da estação de monta reprodutiva. Porém, o endométrio com esses critérios de uma égua que está infértil dois ou mais anos é classificado na Categoria IIB. O IFE é de 50 a $80 \%$. Já na Categoria IIB, estão classificadas aquelas éguas com mais de uma das alterações especificadas na Categoria IIA ou com focos inflamatórios, disseminados, difusos, moderados a acentuados; com fibrose de ramos glandulares individuais disseminada e distribuída uniformemente ou com uma média de dois a quatro ninhos fibróticos por $5,5 \mathrm{~mm}$ de campo linear em quatro ou mais campos. O IFE é de 10 a 50\%. Finalmente, na Categoria III, são caracterizadas éguas com mais de uma das alterações especificadas na Categoria IIB ou com alterações inflamatórias difusas, disseminadas e severas; fibrose de ramos glandulares uniformemente disseminados com cinco ou mais ninhos fibróticos por $5,5 \mathrm{~mm}$ por campo linear; lacunas linfáticas muito severas ou atrofia endometrial durante a estação de monta. O IFE é de 10\%. Porém, a égua não deve ser considerada infértil.

Análise estatística. A associação entre variáveis foi investigada por meio do teste do qui-quadrado e o teste exato de Fisher. O coeficiente Phi (j) foi utilizado para medir a intensidade das associações estatisticamente significativas. A correlação entre duas variáveis dicotômicas, ou seja, cuja modalidade de resposta seja "sim/não", é chamada de coeficiente Phi (j) (Souza 2004). Considerou-se um nível de significância de $5 \%$. A comparação entre médias foi realizada com o teste T-Student. O diagrama de caixa (box plot) descreveu a distribuição das idades pelas categorias de biópsias.

\section{RESULTADOS}

As concentrações plasmáticas de $\mathrm{P} 4$ estão apresentadas no Quadro 1. Apenas duas doadoras sadias em fase de diestro foram investigadas. A concentração média de P4 destas fêmeas foi de $11,25 \pm 2,34 \mathrm{ng} / \mathrm{mL}$. A média da concentração de P4 das doadoras com histórico de subfertilidade na fase de diestro, entre os dias 8 e 12 após a ovulação, foi de $7,89 \pm 7,72 \mathrm{ng} / \mathrm{mL}$. Comparando a média dos dois grupos, doadoras sadias e repetidoras coletadas na fase diestro, não houve diferença estatisticamente significativa entre elas $(p>0,05)$. A concentração média de $\mathrm{P} 4$ em éguas futuras receptoras sadias na fase de diestro, coletadas entre os dias 9 e 12 pós-ovulação foi de $4,89 \pm 3,63 \mathrm{ng} / \mathrm{mL}$ A média da concentração de $\mathrm{P} 4$ verificada nas éguas com histórico de subfertilidade, entre os dias 2 e 12 pósovulação, foi de $6,23 \pm 8,17 \mathrm{ng} / \mathrm{mL}$. Além disso, não observou-se concentrações menores de P4 em éguas mais velhas. Não houve diferença estatística significativa entre as médias das doadoras e receptoras, ambas repetidoras de cio e coletadas na 
Quadro 1. Concentrações plasmáticas de P4 em éguas doadoras e receptoras de embriões em fase de diestro com e sem histórico de subfertilidade

\begin{tabular}{cccc}
\hline Categoria & $\begin{array}{c}\text { Idade } \\
\text { (anos) }\end{array}$ & $\begin{array}{c}\text { Coleta } \\
\text { (dia do } \\
\text { ciclo) }\end{array}$ & $\begin{array}{r}\text { Progester } \\
(\mathrm{ng} / \mathrm{mL})\end{array}$ \\
\hline $\begin{array}{c}\text { Éguas doadoras }^{\text {sadias }}{ }^{\mathrm{a}} \\
1\end{array}$ & & & \\
2 & 7 & D8/9 & 12,90 \\
& 7 & D8 & 9,59 \\
Éguas doadoras $_{\text {repetidoras de cio }}{ }^{\mathrm{b}}$ & & & \\
1 & & & \\
2 & \pm 10 & D8 & 0,89 \\
3 & \pm 18 & D8 & 0,13 \\
4 & 15 & D9 & 3,34 \\
5 & 13 & D10 & 19,68 \\
6 & 12 & D10 & 3,14 \\
7 & 4 & D11 & 3,27 \\
8 & \pm 10 & D8 & 1,87 \\
9 & 23 & D8 & 17,59 \\
10 & 21 & D9 & 12,84 \\
& 20 & D8 & 16,15
\end{tabular}

Éguas receptoras

sadias $^{\mathrm{C}}$

$\begin{array}{lccc}1 & \pm 6 & \text { D9 } & 0,48 \\ 2 & \pm 7 & \text { D9 } & 6,20 \\ 3 & \pm 10 & \text { D12 } & 11,66 \\ 4 & \pm 7 & \text { D10 } & 3,42 \\ 5 & \pm 4 & \text { D9 } & 4,02 \\ 6 & \pm 3 & \text { D10 } & 2,18 \\ 7 & \pm 4 & \text { D9 } & 6,30\end{array}$

Éguas receptoras e repetidoras de cio

$\begin{array}{cccc}1 & \pm 10 & \text { D12 } & 0,1 \\ 2 & \pm 7 & \text { D3 } & 2,37 \\ 3 & \pm 6 & \text { D2 } & 1,20 \\ 4 & 19 & \text { D2 } & 1,01 \\ 5 & 10 & \text { D5 } & 12,90 \\ 6 & 12 & \text { D7 } & 19,81\end{array}$

a Obtenção de embrião em todos os últimos três ciclos (no mínimo).

b Não obtenção de embrião em cada um dos últimos três ciclos $(<3$ embriões consecutivos).

${ }^{c}$ Gestação na estação de monta passada e ausência de alterações relevantes para a reprodução na avaliação ginecológica através da palpação e ultrasonografia.

d Sem confirmação de gestação após três transferências de embrião consecutivas.

fase de diestro $(p>0,05)$. 0 teste exato de Fisher indica evidência de independência entre a concentração de $\mathrm{P} 4 \mathrm{e}$ a subfertilidade das éguas ( $\mathrm{p}=1$; bilateral). Assim, a concentração de P4 não influenciou o estado de subfertilidade das éguas.

$\mathrm{Na}$ avaliação histopatológica, das 11 éguas sem histórico de subfertilidade (doadoras e receptoras), 8 (73\%) tiveram classificação da biópsia endometrial de IIA (Fig.1), 2 (18\%) de IIB (Fig.2) e 1 (9\%) de III (Quadro 2). Outros achados histopatológicos observados foram congestão, edema discreto e áreas de hemorragia. A alteração mais freqüiente foi o infiltrado inflamatório predominantemente mononuclear, endometrite,

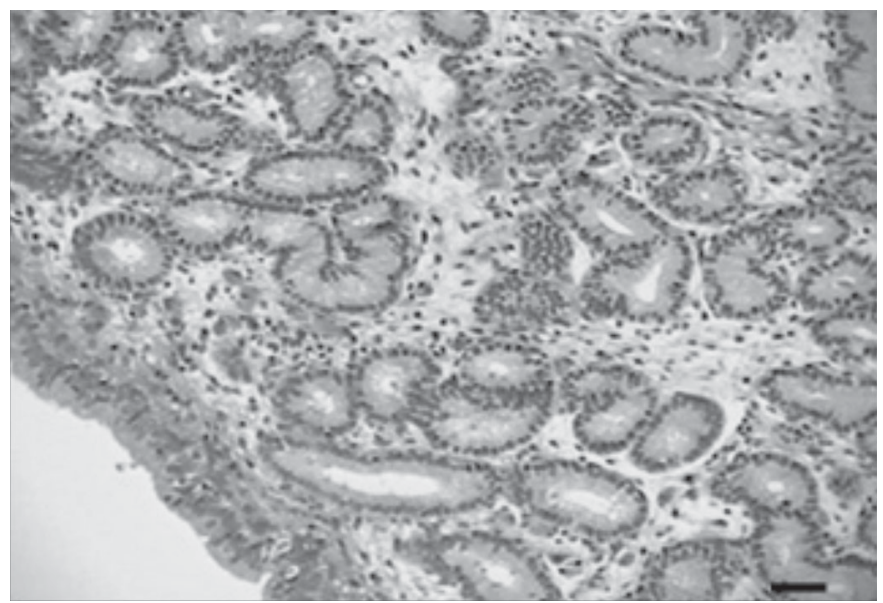

Fig.1. Biópsia endometrial eqüina. Endometrite. Categoria IIA. Animal 2 (égua doadora sadia), áreas de infiltrado inflamatório mononuclear discreto e áreas de edema com hemorragia e congestão. HE, Barra: $100 \mu \mathrm{m}$.

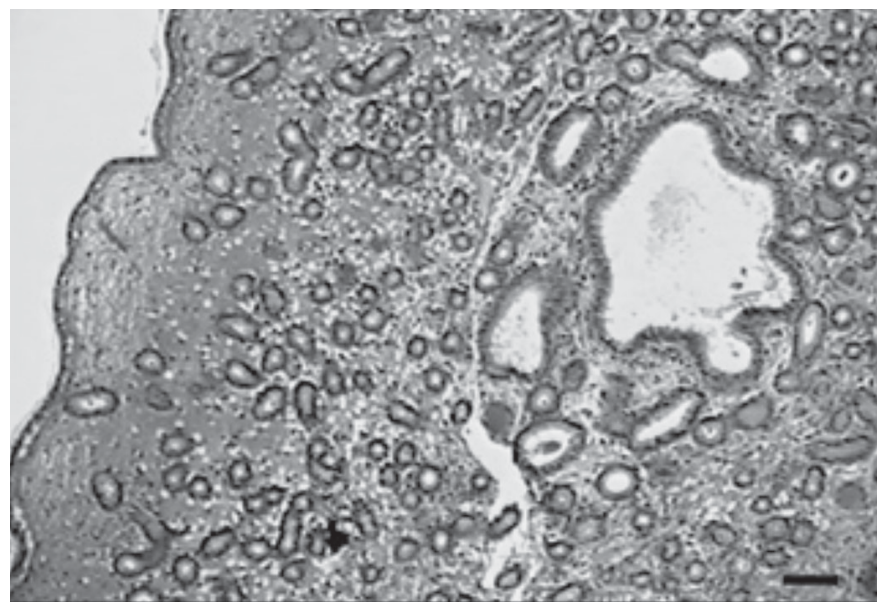

Fig.2. Biópsia endometrial eqüina. Endometrite. Categoria IIB. Animal 4 (égua doadora sadia), edema moderado, congestão e glândulas dilatadas. HE, Barra: $100 \mu \mathrm{m}$.

observado em todas as amostras, onde na maioria mostravase de forma discreta. Nas amostras analisadas, $64 \%$ tiveram endometrite crônica, $27 \%$ endometrite aguda e $9 \%$ endometrite com resposta crônica com uma reação aguda se sobrepondo. $\mathrm{O}$ segundo achado histopatológico mais frequiente foi fibrose, proliferação de tecido conjuntivo, porém bem menos freqüiente que a anterior, em $36 \%$ das amostras. Todas as 25 éguas com histórico de subfertilidade (doadoras e receptoras) apresentaram alguma alteração histopatológica, mesmo que discreta. Das amostras analisadas, $48 \%$ foram classificadas como IIB, $40 \%$ como IIA e $12 \%$ como III (Fig.3-4, Quadro 2). As alterações mais freqüientes observadas foram fibrose periglandular e endometrite aguda e/ou crônica. Não foi observada apenas fibrose em nenhuma amostra.

Em relação às alterações histopatológicas, tanto as éguas consideradas sadias quanto as repetidoras de cio, apresentaram a endometrite e a fibrose como alterações mais freqüentes e estas estiveram sempre presentes, combinadas ou não, em ambos os grupos. Das 8 éguas velhas, $100 \%$ tinham histó- 


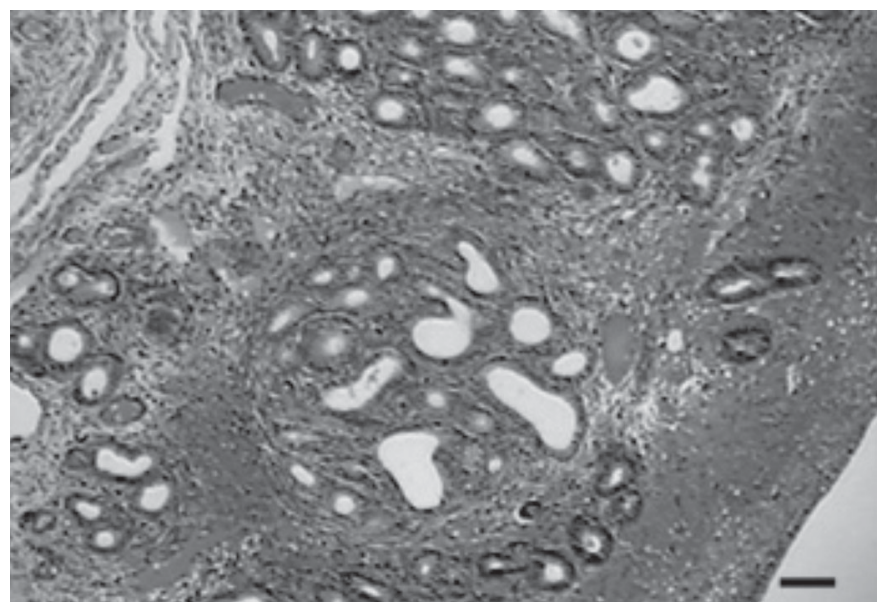

Fig.3. Biópsia endometrial equiina. Endometrite. Categoria III. Animal 3 (égua doadora repetidora de cio), glândulas dilatadas e ninho glandular. HE, Barra: $100 \mu \mathrm{m}$.

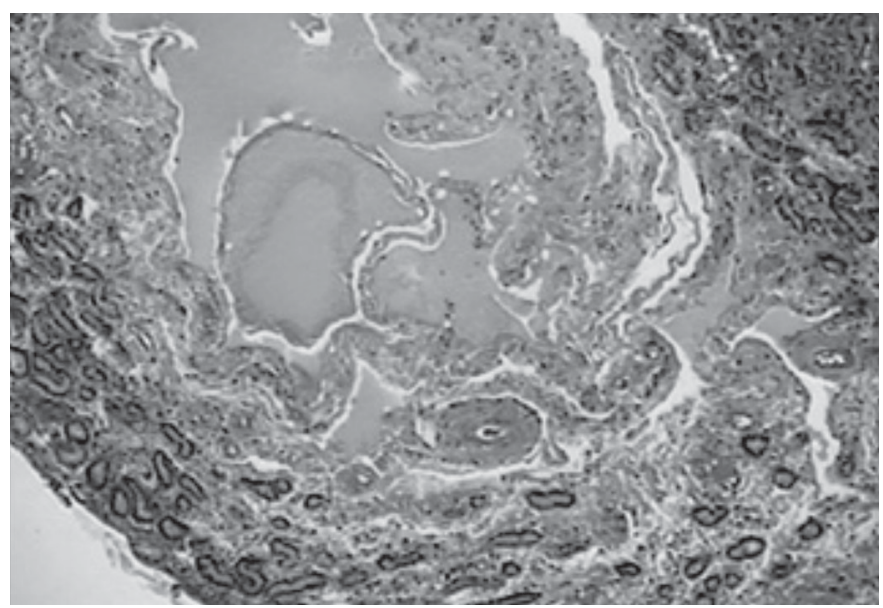

Fig.4. Biópsia endometrial equiina. Endometrite. Categoria III. Animal 17 (égua receptora repetidora de cio), lacunas linfáticas. HE, Barra: $100 \mu \mathrm{m}$.

rico de subfertilidade e das 28 éguas novas, $61 \%$. 0 teste exato de Fisher indica associação estatisticamente significativa entre as duas classificações no sentido de quanto mais velha a égua maior a probabilidade que a mesma seja subfértil $(p=0,036$; unilateral). $O$ grau de associação entre as duas variáveis $(j=-0,355)$ indica uma fraca correlação entre ambas, embora estatisticamente significativa $(p=0,033)(p<0,05)$. Em relação à idade da égua e a categoria da biópsia, segundo o diagrama de caixa, há correlação entre estas duas variáveis, ou seja, quanto mais velha a égua mais chances de terem o endométrio com mais alterações histológicas.

Das 25 éguas com histórico de subfertilidade, 64\% foram classificadas como IIB ou III e 36\% como IIA e das 11 éguas sadias, $73 \%$ foram classificadas como IIA e $27 \%$ como IIB ou III. Nesse sentido, o teste do qui-quadrado indica associação estatisticamente significativa entre a categoria da biópsia endometrial e a subfertilidade das éguas $\left(c^{2}=4,134 ; p=0,042\right.$; bilateral). $O$ grau de associação entre as duas variáveis $(j=-0,339)$ indica uma fraca correlação entre ambas, embora estatistica-
Quadro 2. Classificação da biópsia endometrial, segundo Kenney e Doig (1986), de éguas com e sem histórico de subfertilidade

\begin{tabular}{ccc}
\hline Categoria & $\begin{array}{c}\text { Idade } \\
\text { (anos) }\end{array}$ & $\begin{array}{c}\text { Histopatologia } \\
\text { (categoria) }\end{array}$ \\
\hline
\end{tabular}

Éguas doadoras sadias ${ }^{\mathrm{a}}$

\begin{tabular}{ccc}
1 & 10 & IIA \\
2 & 7 & IIA \\
3 & 7 & IIB \\
4 & 14 & IIB \\
Éguas receptoras sadias $^{\text {b }}$ & & \\
5 & 6 & IIA \\
6 & 7 & IIA \\
7 & 10 & IIA \\
8 & 7 & IIA \\
9 & 4 & III \\
10 & 3 & IIA \\
11 & 4 & IIA \\
Éguas doadoras $_{\text {repetidoras de cio }}{ }^{\text {c }}$ & & \\
1 & & IIB \\
2 & 15 & IIB \\
3 & 10 & III \\
4 & 18 & IIA \\
5 & 4 & IIA \\
6 & 13 & IIB \\
7 & 12 & IIB \\
8 & 4 & III \\
9 & 10 & IIB \\
10 & 23 & IIB \\
11 & 21 & IIB \\
12 & 20 & IIA \\
13 & 10 & III \\
14 & 20 & IIB \\
15 & 10 & IIB \\
\hline & 15 &
\end{tabular}

Éguas receptoras

repetidoras de cio $^{\mathrm{d}}$

$\begin{array}{lcc}16 & 7 & \text { IIB } \\ 17 & 6 & \text { III } \\ 18 & 19 & \text { IIB } \\ 19 & 12 & \text { IIA } \\ 20 & 10 & \text { IIA } \\ 21 & 10 & \text { IIB } \\ 22 & 7 & \text { IIA } \\ 23 & 12 & \text { IIA } \\ 24 & 7 & \text { IIA } \\ 25 & 11 & \text { IIA }\end{array}$

a Obtenção de embrião em todos os últimos três ciclos (no mínimo).

b Não obtenção de embrião em cada um dos últimos três ciclos $(<3$ embriões consecutivos)

c Gestação na estação de monta passada e ausência de alterações relevantes para a reprodução na avaliação ginecológica através da palpação e ultrasonografia.

d Sem confirmação de gestação após três transferências de embrião consecutivas.

mente significativa $(p=0,042)(p<0,05)$. 0 teste exato de Fisher permite concluir, com significância estatística, que o enquadramento na categoria de biópsia com mais alterações histológicas se associa ao estado de subfertilidade $(p=0,047)$ $(\mathrm{p}<0,05)$.

\section{DISCUSSÃO}

A P4 é um hormônio importante para a manutenção da gestação (Cooneely 2001). Valores abaixo de $1,0 \mathrm{ng} / \mathrm{mL}$ normalmen- 
te são insuficientes para manter a gestação e o animal pode apresentar morte embrionária como foi observado por Ball \& Daels (1997). Os mesmos autores sugerem que concentrações acima de $2,0 \mathrm{ng} / \mathrm{mL}$ seriam suficientes para a manutenção da gestação. No experimento de Castro (2005) apenas duas éguas tiveram morte embrionária e ambas apresentaram concentração de P4 abaixo de 1,0ng/mL. Porém, segundo Ferreira (2000) e Castro (2005), concentrações de P4 abaixo de 1,0ng/ $\mathrm{mL}$ por um intervalo curto de tempo, de alguns dias, podem nem sempre estar associadas à mortes embrionárias.

As informações sobre a relação entre ecotextura e morfologia do corpo lúteo $(\mathrm{CL})$ e a concentração plasmática de P4 são controversas. Pierson e Ginther (1985) citam que o tamanho e a ecogenicidade luteal podem refletir na produção de P4. Entretanto, Townson et al. (1989), Ferreira (2000), Nagy et al. (2004) e Castro (2005) verificaram que o tamanho do CL parece não ter efeito significativo sobre a concentração plasmática de P4. No presente estudo não foi realizada tal avaliação.

Na fase de diestro, as duas éguas doadoras sadias tiveram concentração de P4 acima de $2,0 \mathrm{ng} / \mathrm{mL}$. No grupo das doadoras subférteis, duas apresentaram baixas concentrações de P4 (0,89 e $0,13 \mathrm{ng} / \mathrm{mL})$, enquanto as demais (8 éguas), apresentaram concentrações consideradas normais para a fase fisiológica na qual se encontravam. No grupo das receptoras sadias, apenas uma apresentou baixa concentração de P4 $(0,48 \mathrm{ng}$ ) $\mathrm{mL}$ ). No grupo das receptoras subférteis três éguas apresentaram baixas concentrações de P4 baixa $(0,1 ; 1,20$ e $1,01 \mathrm{ng} /$ $\mathrm{mL}$ ) sendo que as duas últimas estavam no segundo dia pósovulação. Normalmente nesta fase o CL ainda apresenta baixa síntese de P4 (Aupperle et al. 2000, Arruda et al. 2001, Nagy et al. 2004). As demais éguas apresentaram concentrações acima de 2,0ng/mL, o que segundo Ball \& Daels (1997) têm sido sugeridas como suficientes para a manutenção da gestação.Embora a P4 elevada na fase estrogênica e baixa na fase progesterônica possam determinar respectivamente falhas na ovulação ou ambiente uterino inadequado para a manutenção de gestação (Castro 2005), sendo causas de infertilidade em éguas, neste experimento tais fatos não foram observados. Verifica-se que outros fatores que não hormonais podem ter sido as causas de falhas reprodutivas observadas nas éguas em programas de TE (Mattos 1989, Lopate \& Threlfall 1991).

Quanto à avaliação histopatológica, Papa et al. (1998) observaram que $100 \%$ das éguas com histórico de subfertilidade apresentaram evidências histopatológicas endometriais. Já Ricketts (1975a) encontrou em 93,3\% das éguas e Ricketts (1975b) e Doig et al. (1981) em 90\%. Assim, pressupõe-se a importância do endométrio estar isento de anormalidades para a manutenção da gestação (Kenney 1978). No presente estudo, assim como Doig et al. (1981) e Papa et al. (1998), os achados mais freqüientes foram fibrose e endometrite aguda e/ou crônica. Todas as éguas sem histórico de subfertilidade que foram colocadas neste grupo, neste experimento, mesmo não tendo sido escolhidas em função da idade, eram jovens, a média de idade foi de 7 anos, formando então um grupo de éguas sadias com bom histórico reprodutivo. Sabese que éguas mais velhas geralmente apresentam complica- ções histológicas mais graves do que éguas mais jovens (Queiroz 1991, Ricketts \& Alonso 1991, Carnevale 1992).

A idade da égua é um fator importante considerando que há uma perda maior da gestação com o avançar da idade (Barbacini 1999, Ginther 1985). Aparentemente, este grupo de animais tem sido caracterizado por alterações degenerativas no endométrio (Ball et al. 1987), o que pode dificultar a atividade dos hormônios circulantes, alterar o aporte de células à luz do útero e dificultar a drenagem linfática (Schoon et al. 1997). Essas alterações incluem endometrites, endometrioses e alterações vasculares (Ball et al. 1987). Outra característica é o posicionamento do útero, que nestas éguas está projetado para o interior da cavidade abdominal, apresenta uma angulação maior e um nível mais baixo em relação ao assoalho da pelve, se comparado com o de éguas jovens e sadias. Esta posição dificulta a drenagem do conteúdo uterino (Leblanc et al. 1998). Ainda, a maioria destas éguas apresenta deficiência no fechamento vulvar (Troedsson 1997). Além disso, a sobrevivência dos embriões de éguas senis é significantemente menor do que daqueles provenientes de éguas jovens, provavelmente pelo seu ambiente útero-tubárico e por defeitos no embrião (Ball et al. 1989).

A maioria das éguas do grupo sadias foi classificada como IIA (73\%). Porém duas tiveram resultados dentro da Categoria IIB e uma na Categoria III. Já no grupo das éguas com histórico de subfertilidade, como era de se esperar, a maioria foi classificada como IIB (48\%) e $12 \%$ como III. Porém, houve um número grande de éguas na Categoria IIA (40\%). Isto pode ocorrer porque as causas da subfertilidade podem ser, além das uterinas, intrínsecas como a idade, ovarianas, cervicais e vulvovaginais e/ou extrínsecas como detecção do cio, momento da cobertura, seleção e garanhão selecionado (Mattos 1989), habilidade do técnico, o método de transferência de embriões, a qualidade do embrião transferido, a sincronia da ovulação entre doadora e receptora (Arruda et al. 2001).

\section{CONCLUSÕES}

Conclui-se que as éguas repetidoras apresentam alterações histológicas mais severas do que as classificadas como sadias sendo as endometrites e as fibroses as alterações mais freqüentes. Porém, nem sempre as éguas com alterações muito leves a moderadas levam uma gestação a termo e nem sempre as com alterações severas falham na reprodução. Por outro lado, a concentração de progesterona e a subfertilidade parecem ser variáveis independentes. Em relação a idade, as éguas velhas têm mais chance de apresentarem subfertilidade. Sugere-se que a biópsia endometrial é uma técnica importante para a avaliação da fertilidade da égua, além de ser fácil, segura, barata e com um desconforto mínimo para o animal.

Agradecimentos.- Os autores agradecem ao curso de Pós-Graduação de Clínica e Reprodução Animal, Universidade Federal Fluminense, e ao CNPq pelo suporte financeiro.

\section{REFERÊNCIAS}

Aupperle H., Özgen S., Schoon H.A., Schoon D., Hoppen H.O., Sieme H. \& Tannapfel A. 2000. Cyclical endometrial steroid hormone receptor 
expression and proliferation intensity in the mare. Equine Vet. J. 32(3):228232.

Arruda R.P., Visintin J.A., Fleury J.J., Garcia A.R., Madureira E.H., Celeghini E.C.C. \& Neves Neto J.R. 2001. Existem relações entre tamanho e morfoecogenicidade do corpo lúteo detectados pelo ultra-som e os teores de progesterona plasmática em receptoras de embriões eqüinos? Braz. J. Vet. Res. Anim. Sci. 38(5):233-239.

Ball B.A., Hillman R.B. \& Woods G.L. 1987. Survival of equine embryos transferred to normal and subfertile mares. Theriogenology 28(2):167-174.

Ball B.A. \& Daels P.F. 1997. Early pregnancy loss in mares: applications for progestin therapy, p.531-534. In: Robinson N.E. (ed.), Current Therapy in Equine Medicine. 4th ed. W.B. Saunders, Philadelphia.

Barbacini S., Gulden P., Marchi V. \& Zavaglia G. 1999. Incidence of embryo loss in mares inseminated before and after ovulation. Equine Vet. Education. 10:108-111.

Carnevale E.M. \& Ginther O.J. 1992. Relationships of age to uterine function and reproductive efficiency in mares. Theriogenology 37(5):1101-1115.

Castro R.P.R. 2005. Influência de aspectos reprodutivos e hormonais de éguas doadoras e receptoras de embriões da raça Campolina sobre a taxa de gestação e morte embrionária. Dissertação de Mestrado em Reprodução, Programa de Pós-Graduação em Medicina Veterinária, Universidade Federal Fluminense, Niterói, RJ. 70p.

Concha-Bermejillo A. \& Kennedy P.C. 1982. Prognostic value of endometrial biopsy in the mare: a retrospective analysis. J. Am. Vet. Med. Assoc. 181(7):680-681.

Cooneely O.M. 2001. Perspective: Female steroid hormone action. Endocrinology 142(6):2194-2199.

Doig P.A., McNight J.D. \& Miller R.B. 1981. The use of endometrial biopsy in the infertile mare. Can. Vet. J. 22:72-76.

Evans M.J., Hamer J.M., Gason L.M. \& Irvine A.C. 1987. Factors affecting uterine clearance of inoculated materials in mares. J. Reprod. Fertil. 35 (Suppl.):327342.

Ferreira J.B.P. 2000. Características ultra-sonográficas e concentração de progesterona na ocorrência de morte embrionária em éguas (Equus caballus) da raça Campolina. Dissertação de Mestrado em Reprodução, Programa de Pós-Graduação em Medicina Veterinária, Universidade Federal Fluminense, Niterói, RJ. 81p.

Ginther O.J. 1985. Embryonic loss in mares: incidence, time of occurrence and hormonal involvement. Theriogenology 23(1):77-89.

Jones D.M., Fielden E.D. \& Carr D.H. 1991. Some physiological and pharmacological factor affecting uterine motility as measured by electromyography in the mare. J. Reprod. Fertil. 44 (Suppl.):357-358.

Junqueira L.C.U. \& Carneiro J. 1999. Histologia Básica. 9a ed. Guanabara Koogan, Rio de Janeiro.

Kenney R.M. 1975. Prognostic value of endometrial biopsy of the mare. J. Reprod. Fertil. 23:347-348.

Kenney R.M. 1978. Cyclic and pathologic changes of the mare endometrium as detected by biopsy, with a note on early embryonic death. J. Am. Vet. Med. Assoc. 172(3):241-262.
Kenney R.M. \& Doig P.A. 1986. Equine endometrial biopsy, p.723-29. In: Morrow D.A. (ed.), Current theraphy in theriogenology: diagnosis, treatment and prevention of reproductive diseases in small and large animals. Vol.2. W.B. Saunders, Philadelphia.

LeBlanc M.M., Neuwirth L., Jones L., Cage C. \& Mauragis D. 1998. Differences in uterine position of reproductively normal mares and those with delayed uterine clearance detected by scintigraphy. Theriogenology 50:49-54.

Lopate C. \& Threlfall W.R. 1991. Assessment of luteal function with progesterone enzyme immunoassays in the horse mare. Theriogenology 35(3):583-590.

Mattos R.C. 1989. Manejo reprodutivo da égua, p.10-15. In: Taranto J.R. (ed.), Sangue e Raça: o cavalo de corrida brasileiro. Index, Rio de Janeiro.

Nagy P., Huszenicza G., Reiczigel J., Juhász J., Kulcsár M., Abaváry K. \& Guillaume D. 2004. Factors affecting plasma progesterone concentration and the retrospective determination of time of ovulation in cyclic mares. Theriogenology 61:203-214.

Papa F.O., Lopes M.D., Alvarenga M.A., Meira C., Luvizzoto M.C.R., Langoni H., Ribeiro E.F., Azedo A.E. \& Bonfim A.C.M. 1998. Early embryonic death in mares: clinical and hormonal aspects. Braz. J. Vet. Res. Anim. Sci. 35(4):170-173.

Pierson R.A. \& Ginther O.J. 1985. Ultrasonic evaluation of the Corpus luteum of the mare. Theriogenology 23(5):795-806.

Queiroz F.J.R. 1991. Biópsia endometrial como método auxiliar de diagnóstico da subfertilidade e da infertilidade na égua (Equus cabalus, L. 1728). Dissertação de Mestrado em Reprodução, Programa de Pós-Graduação em Medicina Veterinária, Universidade Federal Fluminense, Niterói, RJ. 75p.

Ricketts S.W. \& Alonso S. 1991. The effect of age and parity on the development of equine chronic endometrial disease. Equine Vet. J. 23(3): 189-192.

Ricketts S.W. 1975a. The technique and clinical application of endometrial biopsy in the mare. Equine Vet. J. 7(2):102-108.

Ricketts S.W. 1975b. Endometrial biopsy as a guide to diagnosis of endometrial pathology in the mare. J. Reprod. Fertil. 23(Suppl.):341-345.

Schoon H.A., Schoon D. \& Klug E. 1997. Vascular lesions in the equine endometrium. Pferdeheilkunde 13:546.

Silva C.A.M., Barros S.S., Esquerre R.A. \& Silva J.H.S. 1987. A biópsia endometrial na avaliação da fertilidade na égua. Pesq. Vet. Bras. 7(4):131133.

Souza A.C. 2004. A relação entre a teoria clássica de medida e teoria da resposta ao item. Trabalho de conclusão de curso, apresentado para obtenção de grau de Bacharel em Estatística, Colegiado de Curso de Bacharelado em Estatística, Universidade Federal do Pará, Belém. 52p.

Thownson D.H., Pierson R.A. \& Ginther O.J. 1989. Characterization of plasma progesterone concentration for two distinct luteal morphologies in mares. Theriogenology 32(2):197-204.

Troedsson M.H.T. 1997. Therapeutic consideration for mating-induced endometritis. Pferdeheilkunde 13:516-520.

Van Camp S.D. 1988. Endometrial biopsy of the mare: a review and update. Vet. Clin. North Am. Equine Pract. 4(2):229-245. 